\title{
ARTICLE
}

\section{Neutron dose equivalent rates in the vicinity of waste containers containing TRU}

\author{
Nolan E. Hertel ${ }^{\mathrm{a}^{*}}$, Taiee Liang ${ }^{\mathrm{a}}$, Timothy Cahill ${ }^{\mathrm{a}}$, Michael L. Littleton ${ }^{\mathrm{b}}$, Scott A. Byers ${ }^{\mathrm{b}}$ and Eric A. Burgett ${ }^{\mathrm{c}}$ \\ ${ }^{a}$ Nuclear and Radiological Engineering Program, G. W. Woodruff School of Mechanical Engineering, Georgia Institute of \\ Technology, Atlanta, GA 30332-0745, USA ${ }^{b}$ Wastren Advantage, Inc., Transuranic Waste Processing Facility, 100 WIPP Road, \\ Lenoir City, TN 37771, USA; ${ }^{c}$ Department of Nuclear Engineering and Health Physics, Idaho State University, Pocatello, ID \\ 83209-8060, USA
}

\begin{abstract}
The Transuranic Waste Processing Center is charged with treating, packaging and shipping TRU waste for disposal in a safe and efficient manner. The nature of TRU waste, i.e. it contains radionuclides which spontaneous fission and emit alpha particles which can lead to the production of $(\alpha, n)$ neutrons, can lead to neutron dose equivalent rates of radiation protection concern. Neutron dosimetry measurements were performed at the TWPC facility in locations where neutron dose equivalent rates of significance had been observed using neutron survey instruments. These measurements provide an assessment of the dosimetric properties of neutron fields that are deemed to be typical at the TWPC. The neutron measurements were performed at four locations using a Bonner sphere spectrometer which provides a low resolution spectrum that yields a good estimate of the neutron dose equivalent rate and the fluence rate. A tissue equivalent proportional counter dose equivalent measurement was performed at one location. Based on the Bonner sphere measurements, over $90 \%$ of the ambient dose equivalent is delivered between approximately $100 \mathrm{keV}$ and 7.4 MeV.
\end{abstract}

\section{Keywords: TRU waste; Neutron dose equivalent rates; Bonner sphere spectrometer; neutron fluence rate distributions}

\section{Introduction}

The Transuranic (TRU) Waste Processing Center (TWPC) in Lenoir City, Tennessee is charged with treating, packaging and shipping TRU waste for disposal in a safe and efficient manner. The nature of TRU waste, i.e. the spontaneous fission of ${ }^{252} \mathrm{Cf}$ and ${ }^{244} \mathrm{Cm}$ and the potential for producing $(\alpha, n)$ neutrons from the $\alpha$ decay of TRU radionuclides, results in neutron dose equivalent rates of significance for radiation protection. Neutron dosimetry measurements were performed at four locations at the TWPC. These measurements provided an independent assessment of the dosimetric properties of the neutron fields that are deemed to be typical of the neutron fields at the TWPC and were performed at locations where neutron dose equivalent rates of significance were previously observed.

The measurements were performed at these four locations using a Bonner sphere spectrometer (BSS). The BSS provides a low resolution spectrum that yields a good estimate of the neutron dose equivalent rate and the total fluence rate. The BSS set employed in these measurements has been used at other facilities to provide similar neutron field characterization. A REM-500

*Corresponding author. Email: nolan.hertel@me.gatech.edu tissue equivalent proportional counter (TEPC) was used to perform a measurement at one location as well.

\section{Equipment}

\subsection{Bonner sphere spectrometer}

The Ludlum BSS system used for the measurements is essentially the same design as the original system reported by Bramblett, Ewing and Bonner.[1] It consists of a $4 \mathrm{~mm} \times 4 \mathrm{~mm} \varnothing, 96.1 \%$-enriched $\mathrm{LiI}(\mathrm{Eu})$ crystal placed inside polyethylene moderating spheres. Neutrons incident on the surface of the polyethylene spheres slow down to low energies at which they have a very high probability of capture via the $(n, \alpha)$ reaction in ${ }^{6} \mathrm{Li}$. The standard BSS measurement set includes irradiation of the bare LiI detector and the detector placed in six different diameter polyethylene spheres, namely, spheres with diameters of 5.08, 7.62, 12.7, $30.32,25.4$, and $30.48 \mathrm{~cm}$.

The endpoint of the BSS measurements is a count rate for each moderator-detector combination. To determine the fluence rate, a matrix equation form of the Fredholm integral equation must be solved and the energy response of each detector. In the present work, the BUMS unfolding code was employed. [2] The BUMS 
code is a web-based unfolding code developed at Georgia Tech that provides several unfolding algorithms from which the user may select. In this work and most work previously done at Georgia Tech, the SPUNIT algorithm was used in the unfolding process.[4] SPUNIT is an adaptation of the method described by Doroschenko [5] and is based on the application of that method in the U.S. Naval Research Laboratory code BUNKI.[6] BUMS features a selection of response matrices; the 31-group UTA4 response matrix created by Johnson [6] from the 171 energy group responses of Hertel and Davidson[3] was used in this work. The software also provides a variety of spectra that can be used as the initial guess in the unfolding procedure.

The BUMS code generates a low resolution neutron multigroup fluence rate as well as the values of several dose equivalent quantities computed with the fluence rate spectra. During the iteration process, detector count rates computed with the current fluence rate are compared with the measured count rates. When the average of the difference between the calculated sphere count rates and the measured count rates is within a user-specified percentage, the iteration process is terminated. If this convergence agreement is not met, the process is terminated when the number of iterations on the fluence rate spectrum reaches an upper limit input by the user. Typically the iteration limit is set at 1000 since over-iteration can occur. This agreement percentage is not an uncertainty estimate of the fluence rate and related data, but a measure of the consistency of the count rate data calculated with the computed fluence rate and the measured count rate data. Although the energy-dependent spectrum from the unfolding exhibits sensitivity to the spectrum used to initiate the iteration process, the total fluence and dose equivalent quantities cluster about a given value for reasonable starting fluence guess.

The Georgia Tech BSS set used in the measurements was calibrated at Georgia Tech using an unmoderated and $\mathrm{D}_{2} \mathrm{O}$-moderated ${ }^{252} \mathrm{Cf}$ neutron field. The resulting calibration factor was and has historically been within $5 \%$ of unity, depending on the method used to correct the measured reference field count rates for room-return and air scatter. For these measurements a fluence calibration factor of unity was used. The BSS set was also been calibrated at the neutron calibration facility at the PTB in 2008 as a part of the calibration of an extended BSS set, lending further credence to its calibration.[7]

\subsection{Tissue-Equivalent Proportional Counter}

For these measurements a Health Physics Instruments REM-500 handheld TEPC detector was used to take data at one location. The TEPC is a sealed spherical counter that is $5.175 \mathrm{~cm}$ in diameter. It is filled with propane gas to simulate 2 microns of tissue (13.3 $\mathrm{mmHg}$ pressure). More information is available in the operating manual. [8] In the present work, the tepc_ng code,[9] developed at Pacific Northwest
National Lab (PNNL) and recently updated by PNNL, was used to obtain the dose equivalent rate from the TEPC pulse-height data. The code makes corrections for the TEPC gas pressure and temperature. The lineal energy calibration is performed by the PNNL code using the "proton drop" edge channel and the neutron start channel. After performing the calibration, the code then calculates the lineal energy distribution, folds it with ICRP Publication 60 and ICRP Publication 21 [10, 11] fluence-to-dose-equivalent conversion coefficients and returns an integrated average dose rate during the measurement. Discussions of such algorithms and use of lineal energy in microdosimetry for radiation protection are numerous and a complete discussion is too lengthy to include, so the interested reader is referred to Ref. [12] and [13].

\section{Measurement setup and procedure}

Measurements were performed at four locations with the BSS. These locations were selected based on radiation protection surveys which indicated the presence of a non-negligible neutron field. For Location \# 1 the detector center was positioned 161.5 $\mathrm{cm}$ above the floor and $50 \mathrm{~cm}$ from the centerline of a 55-gallon waste drum in the Contact Handled Marshaling Building. Measurement Location \#2 was performed in the 30-ton crane bay. The BSS detector center was placed $158 \mathrm{~cm}$ above the floor and $30 \mathrm{~cm}$ from a remote handled waste cask surface; these casks consist of a sheet metal overpack containing a concrete cask with $15.24 \mathrm{~cm}$ thick walls containing the waste. A REM-500 measurement was also performed at this location subsequent to the BSS measurement.

At Location \#3, the BSS measurement was performed at $34 \mathrm{~cm}$ from the midplane of two drums having similar TWPC neutron survey readings in the Contact Handled Staging Area (CHSA). Measurement Location \#4 was also inside the CHSA. The detector center was located $34 \mathrm{~cm}$ from the centerline of a drum (182.5 $\mathrm{cm}$ above the floor).

\section{Measurement results}

The Bonner sphere alpha count rates obtained at the measurement locations were unfolded. Since the BSS is a very low resolution spectrometer, the quantities of principal interest from the BSS measurements are the ambient dose equivalent and the total fluence rates. Since the unfolding code does not provide an error for these quantities determined from the unfolded spectrum, a number of unfoldings were performed using different starting spectra.

Each spectrum was unfolded using multiple starting spectra or initial guesses. There is library of starting spectra available in the BUMS scroll down menu; they are available in the online code, see Ref. [2]. Unfoldings were performed using 15-20 starting spectra from that library, most of which were spectra from 
reactors and various configurations of $\mathrm{PuBe}$ and $\mathrm{Cf}-252$ sources. The unfolded spectra retained for the analysis were those that yielded reasonable results for the neutron field, i.e. had neutrons in the anticipated energy ranges for these measurements (thermal $-15 \mathrm{MeV}$ ) and provided a "good" match to the measured count rates. In general 10 unfolded spectra were selected for each measurement location based on the average of the computed and measured count rate differences. The results for those unfoldings were averaged to provide a mean fluence rate (as well as a fluence rate spectrum), mean energy for the spectrum, and mean dose equivalent rate at that location. The associated standard deviations in these values were computed and are reported with the values in Table 1. The standard deviations in this table do not include the $5 \%$ calibration uncertainty of the Bonner sphere set. The standard deviations indicate that the resulting total fluxes and dose equivalent rates at each location were quite close in value regardless of the starting spectrum used. This lends confidence that the BSS systems are good estimators of dose equivalent and total flux. The resulting neutron fluence rate spectra are shown in Figures 1-4.

The TEPC spectrum at Location \#2 was processed. After processing, an ambient dose equivalent rate, based on ICRP 60 quality factors, of $98 \mu \mathrm{Sv} / \mathrm{hr} \pm 12 \%$ and an average quality factor of 15.32 were obtained.

\section{Conclusions}

In Figure 5, the fraction of the cumulative ambient dose equivalent as a function of energy is plotted for each location using the mean spectra previously described. For all locations, over $90 \%$ of the dose is delivered between approximately $100 \mathrm{keV}$ and $7.4 \mathrm{MeV}$. At Location \#2, approximately $5 \%$ of the dose is delivered by thermal neutrons. This can be observed by the higher thermal neutron flux observed at that location (Figure 2) in comparison to other locations. The higher thermal neutron dose at Location 2 is attributed to the greater thermalization of neutrons by the concrete cask wall. The larger uncertainty at Location 1 in the average energy is largely a result of the fact that the starting spectra which yielded acceptable count rates were more different in shape than for the other locations.

The BSS ambient dose equivalent rate (Table 1) at Location \#2 is approximately $12 \%$ higher than the TEPC determined value. In a statistical sense, it is within one standard deviation of the TEPC results thereby not causing much concern. However, it would be desirable for the TEPC data to have had lower statistical error, particularly to locate the proton drop.

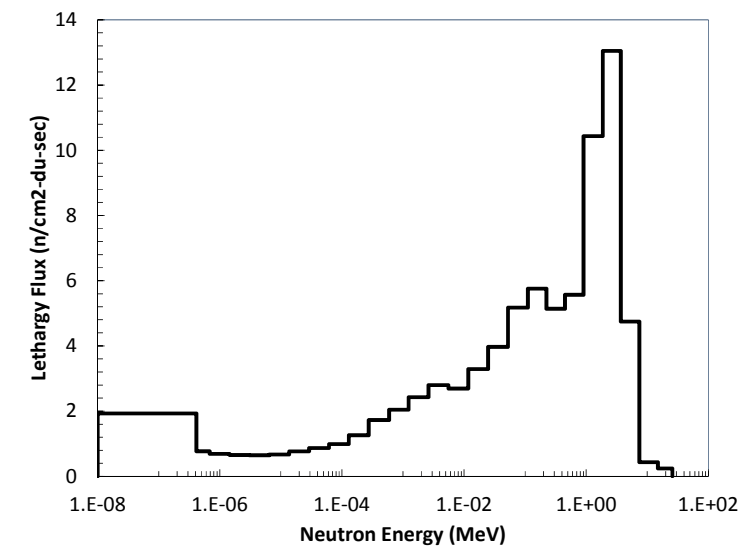

Figure 1. Location \#1 Mean Neutron Lethargy Fluence Rate.

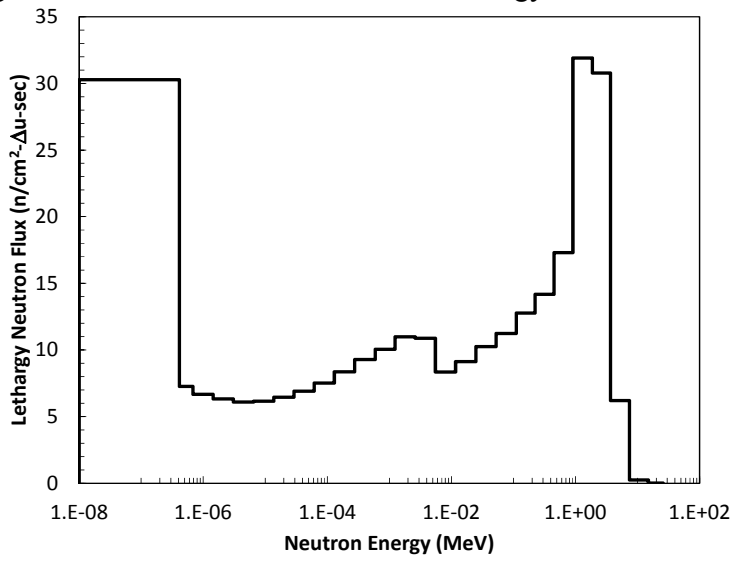

Figure 2. Location \#2 Mean Neutron Lethargy Fluence Rate.

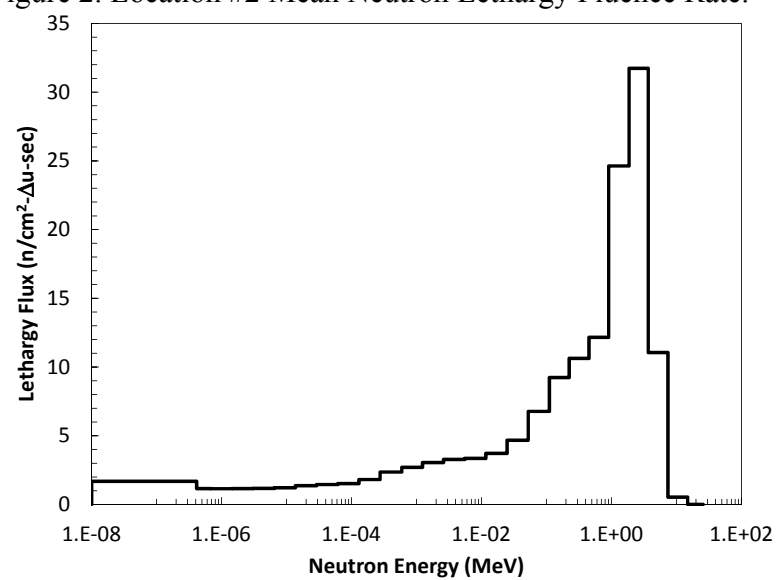

Figure 3. Location \#3 Mean Neutron Lethargy Fluence Rate.

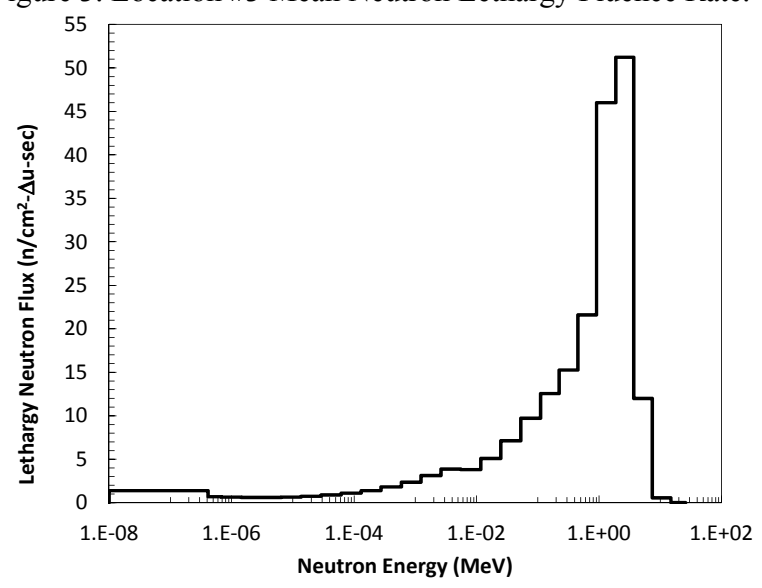

Figure 4. Location \#4 Mean Neutron Lethargy Fluence Rate. 
Table 1. Mean Dosimetric Quantities and their Standard Deviations Obtained from the Bonner Sphere Unfolding.

\begin{tabular}{ccccccc}
\hline Location & $\begin{array}{c}\text { Flux } \\
\left(\mathbf{n} / \mathbf{c m}^{\mathbf{2}} \text {-sec) }\right.\end{array}$ & $\begin{array}{c}\text { Uncertainty } \\
\mathbf{( \% )}\end{array}$ & $\begin{array}{c}\text { Averge Energy } \\
(\mathbf{M e V})\end{array}$ & $\begin{array}{c}\text { Uncertainty } \\
\mathbf{( \% )}\end{array}$ & $\begin{array}{c}\text { Ambient Dose } \\
\text { Equivalent }(\boldsymbol{\mu} \mathbf{S v} / \mathbf{h r})\end{array}$ & $\begin{array}{c}\text { Uncertainty } \\
(\mathbf{\%})\end{array}$ \\
\hline$\# 1$ & 62 & 1.1 & 1.01 & 26.0 & 44 & 0.4 \\
$\# 2$ & 297 & 0.2 & 0.42 & 3.2 & 113 & 0.7 \\
$\# 3$ & 107 & 0.4 & 1.25 & 5.1 & 95 & 0.9 \\
$\# 4$ & 164 & 0.3 & 1.32 & 3.7 & 167 & 0.7 \\
\hline
\end{tabular}

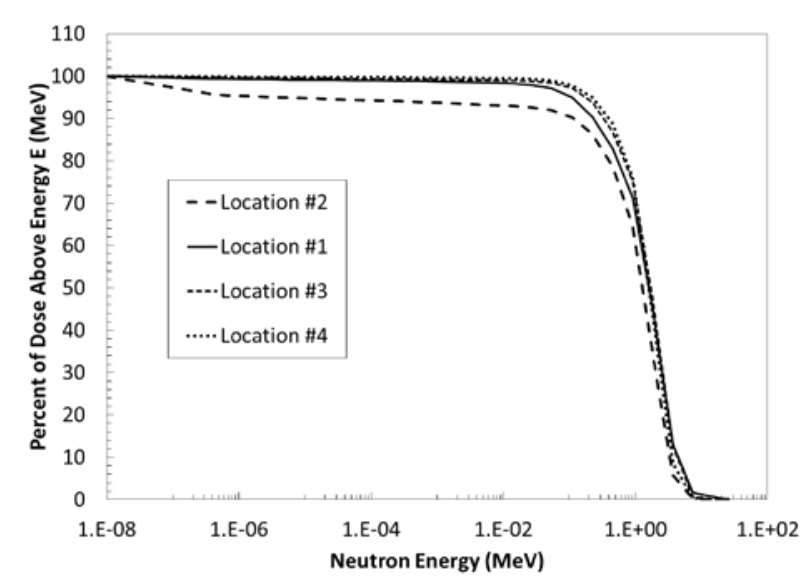

Figure 5. Fraction of Dose Equivalent as a Function of Energy. The ordinate is the fraction of the dose equivalent that is above the corresponding energy.

\section{References}

[1] R. L. Bramblett, R. I. Ewing and T. W. Bonner, A new type of neutron spectrometer, Nuclear Instruments and Methods 9 (1960), pp. 1-12.

[2] J. Sweezy, N. Hertel and K. Veinot, BUMS ${ }^{\mathrm{a}}-$ Bonner sphere unfolding made simple: An HTML multisphere neutron spectrometer unfolding package, Nuclear Instruments and Methods 476 (2002), pp. 263-269.

[3] N. E. Hertel and J. W. Davidson, The response of bonner spheres from thermal energies to $17.3 \mathrm{MeV}$, Nuclear Instruments and Methods A238 (1985), pp. 509-516.

[4] L. W. Brackenbush and R. I. Scherpelz, SPUNIT, A computer code for multisphere unfolding, computer applications in health physics, Proceedings of the Seventeenth Midyear Topical Symposium of the Health Physics Society, Richland,

${ }^{a}$ BUMS Code is located at the following URL:

http://nukeisit.gatech.edu/cgi-bin/notezy_1.2/notezy.cgi
WA (1984).

[5] J. J. Doroshenko, S. N. Kraitor, T. V. Kuznetsova, K. K. Kushnereva and E. S. Leonov, New methods for measuring neutron spectra with energy from $0.4 \mathrm{eV}$ to $10 \mathrm{MeV}$ by track and activation detectors, Nuclear Technology 33 (1977), pp. 296-304.

[6] T. L. Johnson, Y. Lee, K. A. Lowry and S. G. Gorbics, Recent advances in bonner sphere neutron spectrometry, Proceedings Theory and Practices in Radiation Protection and Shielding Symposium, Knoxville, TN (1987), pp. 83-84.

[7] R. M. Howell, E. A. Burgett, B. Wiegel and N. E. Hertel, Calibration of a bonner sphere extension (BSE) for high-energy neutron spectrometer, Radiation Measurements 45 (2010), pp. 1233-1237.

[8] Health Physics Instruments, Operations And Repair Manual, Model REM 500b Neutron Survey Meter, February 2005 Revision B3.

[9] R. Stewart and R. Scherpelz, TEPC Pulse-Height Analysis Software, Pacific Northwest National Laboratory, PNWD-3032.

[10] International Commission on Radiological Protection (ICRU), 1990 Recommendations of the International Commission on Radiological Protection, ICRP Publication 60, Pergamon Press, New York, (1991).

[11] International Commission on Radiological Protection, Data for Protection against Ionizing Radiation from External Sources, ICRP Publication 21, Pergamon Press, Oxford, (1973).

[12]L. W. Brackenbush, J. C. McDonald, G. W. R. Endres and W. Quam, Mixed field dose equivalent measuring instruments, Radiation Protection Dosimetry 10 (1985), pp. 307-318.

[13] International Commission on Radiation Units and Measurements (ICRU), Microdosimetry. ICRU Report No. 36, ICRU, Bethesda, Maryland (1983). 